\title{
Dynamics of Particle in a Box in Time Varying Potential Due to Chirped Laser Pulse
}

\author{
Brijender Dahiya, Vinod Prasad \\ Department of Physics, Swami Shraddhanand College, University of Delhi, Delhi, India \\ E-mail: brijender.dahiya@gmail.com,vprasad@ss.du.ac.in \\ Received August 13, 2010; revised October 15, 2010; accepted November 19, 2010
}

\begin{abstract}
We describe a computational method for simulating the time dependent quantum mechanical system interacting with external field. In this method the Schrödinger equation is solved by expanding the wave function in the basis set of unperturbed Hamiltonian. The expansion yields a set of coupled first order differential equation. For expansion coefficients, the coupled channel method is applied to a particle in a box interacting with external field in the form of chirped laser pulse. The pulse shape is taken as Gaussian. We study the effect of different pulse parameters i.e. chirp rate, intensity, center frequency, box length and laser duration on the dynamics of the particle. Many interesting results are obtained and explained.
\end{abstract}

Keywords: Transition Probability, Chirp Rate, Box Length

\section{Introduction}

Quantum mechanics $[1,2]$ is the fundamental base for several branches in Physics and particle in a box is one of the fundamental problems of quantum mechanics. In this paper, we have studied dynamics of a particle in a box in time varying external field/potential. This time varying field is achieved using a chirped laser pulse. Wang and Champagne [3] have studied the interaction of Gaussian laser pulse with the particle in a box. In their study they found that by using laser centre frequency to be resonant with the transition between first two states i.e. $n=1$ and $n=2$, transition probability of $n=2$ state is appreciable and all other higher state have non zero but negligible probability. Here, we are presenting numerical simulation of the system. We numerically solve the coupled differential equations obtained for a particle in a box, in the presence of a chirped laser pulse. It is shown that high-efficiency population transfer is possible for several values of chirp rate and box length. We also explore the dependence of population transfer on chirp rate. The results indicate that we can have large transition probabilities for higher states also with the help of chirped laser pulse.

With the development in laser technology [4-6] in recent years, various techniques have been developed to modulate and even shape the laser pulse. The most exploited feature of modulated pulse is the chirp which describes the variation of carrier frequency with time. If the frequency in- creases with the time, the pulse is positively chirped and if the frequency decreases with time, it is negatively chirped. Ruhman and Kosloff [7] used negatively chirped pulse to achieve large amplitude of vibrational motion in higher vibrational states of the ground electronic surface of CsI through an effective intra pulse pump dump mechanism. Cao et al. [8] have studied that a positively chirped pulse is very efficient in population inversion. So both positive and negative chirps are important and can be used as per requirement of the problem or the system. The selection also depends on the system with which the laser pulse is interacting and other parameters of pulse e.g. intensity, center frequency, pulse width etc. No work for such type of problem has been published earlier. Recently coherent control for box potential with laser fields has been studied by Imre F. Barna and PéterDombi [9].

In this paper we have used numerical method and computer simulation to express all results. In Section 2, we have described a general coupled channel method to solve the Schrödinger equation under time dependent perturbation condition. The Schrödinger equation is reduced to a set of first order coupled differential equations which are solved using efficient and commonly used fourth order Runge-Kutta method. Section 3 briefly describe particle in a box and its interaction with chirped laser pulse. We apply the coupled channel method to find the solution. In Section 4 , we briefly describe the computer simulation work. Finally in Section 5, we describe the numerical results for the 
transition probabilities for different states as a function of time, chirp rate, box length and laser center frequency. Particularly, effect of linear chirp is examined in detail. In Section 6, we conclude the paper.

\section{The Coupled Channel Method}

The coupled channel method [10,11] is used to solve the time dependent Schrödinger equation. Consider a quantum mechanical system having unperturbed Hamiltonian $\hat{H}_{0}$ for a eigen state $\left|\chi_{n}\right\rangle$ with eigen energy values $E_{n}$ which satisfy the equation

$$
\hat{H}_{0}\left|\chi_{n}\right\rangle=E_{n}\left|\chi_{n}\right\rangle
$$

Here $\left|\chi_{n}\right\rangle$ forms a complete orthogonal set of eigen vectors i.e.

$$
\sum\left\langle\chi_{n} \mid \chi_{n}\right\rangle=1,\left|\chi_{n}\right\rangle\left\langle\chi_{n}\right|=\delta_{m n}
$$

Considering the wave function as

$$
|\psi(t)\rangle=\sum_{n} a_{n}(0) \exp \left(-i E_{n} t / \hbar\right)\left|\chi_{n}\right\rangle
$$

Without perturbation wave function will have usual time dependence but coefficient $a_{n}$ is independent of time. Let us consider that perturbation is turned on instantaneously at $t=0$. Then the full Hamiltonian will be described as

$$
\begin{gathered}
\hat{H}=\hat{H}_{0}+V(t) \\
\hat{H}\left|\chi_{n}\right\rangle=\left(\hat{H}_{0}+V(t)\right)\left|\chi_{n}\right\rangle \\
\sum_{n}\left\{i \hbar \frac{\partial a_{n}(t)}{\partial t} \exp \left(-i E_{n} t / \hbar\right)+E_{n} a_{n}(t) \exp \left(-i E_{n} t / \hbar\right)\right\}\left|\chi_{n}\right\rangle \\
=\sum_{n} a_{n}(t) \exp \left(-i E_{n} t / \hbar\right) E_{n}\left|\chi_{n}\right\rangle+\sum_{n} a_{n}(t) \exp \left(-i E_{n} t / \hbar\right) V\left|\chi_{n}\right\rangle
\end{gathered}
$$

$\boldsymbol{V}(t)$ is the perturbation part (which is a function of time) having condition

$$
\begin{array}{ll}
V(t)=0 & t \leq 0 \\
V(t) \neq 0 & t \geq 0
\end{array}
$$

Now the time dependent Schrödinger equation is (as explained in Section 5, box units are used throughout the paper)

$$
i \hbar \frac{\partial|\psi(t)\rangle}{\partial t}=\hat{H}|\psi(t)\rangle
$$

The solution of "(7)" can be written as

$$
|\psi(t)\rangle=\sum_{n} a_{n}(t) \exp \left(-i E_{n} t / \hbar\right)\left|\chi_{n}\right\rangle
$$

Here $a_{n}$ (probability amplitudes) acquire time dependence. In the absence of external perturbation, if system is in one of the eigen state of $\hat{H}_{0}$, then it will remain in that state forever. However by the presence of a small perturbation, the system makes transition between its unperturbed eigen states. So with the perturbation, the probability of finding the system in $n^{\text {th }}$ state at any time $t$ will vary with time and $P_{n}(t)$ is given by

$$
P_{n}(t)=\left|a_{n}(t)\right|^{2}
$$

Using “(4)" in “(7)"

$$
i \hbar \frac{\partial|\psi(t)\rangle}{\partial t}=\left(\hat{H}_{0}+V(t)\right)|\psi(t)\rangle
$$

Using “(1)" and "(8)" in "(10)", we get the following

Or

$$
\sum_{n} i \hbar \frac{\partial a_{n}(t)}{\partial t} \exp \left(-i E_{n} t / \hbar\right)\left|\chi_{n}\right\rangle=\sum_{n} a_{n}(t) \exp \left(-i E_{n} t / \hbar\right) V\left|\chi_{n}\right\rangle
$$

Operating it with $\left|\chi_{k}\right\rangle$ from left, we get

$$
\begin{gathered}
\sum_{n} i \hbar \frac{\partial a_{n}(t)}{\partial t} \exp \left(-i E_{n} t / \hbar\right)\left\langle\chi_{k} \mid \chi_{n}\right\rangle=\sum_{n} a_{n}(t) \exp \left(-i E_{n} t / \hbar\right)\left\langle\chi_{k}|V| \chi_{n}\right\rangle \\
i \hbar \frac{\partial a_{k}(t)}{\partial t} \exp \left(-i E_{n} t / \hbar\right)=\sum_{n} a_{n}(t) \exp \left(-i E_{n} t / \hbar\right)\left\langle\chi_{k}|V| \chi_{n}\right\rangle
\end{gathered}
$$

Let the transition matrix element be

$$
V_{k n}=\left\langle\chi_{k}|V| \chi_{n}\right\rangle
$$

The transition matrix element $V_{k n}$ may be either zero or non-zero depending upon the selection rules. Considering transition frequency as $\omega_{k n}=\left(E_{k}-E_{n}\right) \hbar$, Equation 
(14) will become

$$
i \hbar \frac{\partial a_{k}(t)}{\partial t}=\sum_{n} a_{n}(t) \exp \left(i \omega_{k n} t\right) V_{k n}
$$

Thus matrix differential equation for co-efficients $a_{n}$ is

$$
i \hbar\left(\begin{array}{c}
\dot{a}_{1} \\
\dot{a}_{2} \\
\cdot \\
\dot{a}_{n}
\end{array}\right)=\left(\begin{array}{cccc}
V_{11} & V_{12} \exp \left(i \omega_{12} t\right) & \cdot & \cdot \\
V_{21} \exp \left(i \omega_{21} t\right) & V_{22} & \cdot & \cdot \\
\cdot & \cdot & \cdot & \cdot \\
\cdot & \cdot & \cdot & V_{n n}
\end{array}\right)\left(\begin{array}{c}
a_{1} \\
a_{2} \\
\cdot \\
a_{n}
\end{array}\right)
$$

And by solving this set of coupled differential equations we get value of $a_{n}(t)$ for different states and hence probability of finding the system in any particular state at any time $t$. We can write

$$
a_{n}(t)=R_{n}(t)+i I_{n}(t)
$$

where $R_{n}(t)$ and $I_{n}(t)$ are real and imaginary part of $a_{n}(t)$ respectively. Using (18) in (16) we can have

$$
\begin{aligned}
& i \hbar\left(\frac{\partial R_{k}(t)}{\partial t}+i \frac{\partial I_{k}(t)}{\partial t}\right)= \\
& \sum_{n}\left(R_{n}(t)+i I_{n}(t)\right)\left(\cos \left(\omega_{k n} t\right)+i \sin \left(\omega_{k n} t\right)\right) V_{k n}
\end{aligned}
$$

Separating real and imaginary parts of $a_{n}(t)$ we get following equations

$$
\begin{gathered}
\hbar \frac{\partial R_{k}(t)}{\partial t}=\sum_{n} R_{n}(t) \sin \left(\omega_{k n} t\right) V_{k n}+I_{n}(t) \cos \left(\omega_{k n} t\right) V_{k n} \\
\hbar \frac{\partial I_{k}(t)}{\partial t}=-\sum_{n} R_{n}(t) \cos \left(\omega_{k n} t\right) V_{k n}+I_{n}(t) \sin \left(\omega_{k n} t\right) V_{k n}
\end{gathered}
$$

Equation (20) and (21) can be solved by using numerical methods e.g. Runge-Kutta Method.

\section{Particle in a Box in Chirped Laser Field}

Consider a particle i.e. electron in a deep potential well from which it cannot escape and loses no energy when it collide with walls. So the potential is defined as

$$
\begin{aligned}
& V(x)=0 \quad 0<x \leq L \\
& V(x)=\infty \quad x<0, x>L
\end{aligned}
$$

Now the Schrödinger equation becomes

$$
-\frac{\hbar^{2}}{2 m} \frac{\partial^{2} \psi(x)}{\partial x^{2}}=E \psi(x)
$$

By solving this equation, the unperturbed eigen function and the eigen energy value are

$$
\psi_{n}(x)=\sqrt{\frac{2}{L}} \sin (n x / L)
$$

and

$$
E_{n}=\hbar^{2} \pi^{2} n^{2} /\left(2 m L^{2}\right)
$$

where $L$ is the length of the box and $n=1,2,3, \cdots$, and $n \neq 0$ as it will give probability of finding the particle everywhere equal to zero, which is not possible. Consider that the system is interacted with chirped laser pulse [12]. Here the laser field is defined as

$$
E(t)=E_{0} \sin ^{2}(\pi t / \tau) \cos \left(\omega_{0}+\beta t\right) t
$$

Here $E_{0}, \tau, \omega_{0}$ and $\beta$ are the amplitude, laser duration, laser center frequency and the chirp rate respectively. The laser interaction with the particle (i.e. electron) is defined as

$$
V(x, t)=e . E(t)(x-L / 2)
$$

Here ' $e$ ' is electron charge and zero point potential is chosen at $L / 2$. Now the transition matrix $V_{k n}$ can be written as

$$
\begin{aligned}
V_{k n}(t) & =\int e E(t) \psi_{k}^{*}(x)(x-L / 2) \psi_{n}(x) d x \\
& =e E(t) \int \psi_{k}^{*}(x)(x-L / 2) \psi_{n}(x) d x \\
& =e E(t) D_{k n}
\end{aligned}
$$

where

$$
\begin{gathered}
D_{k n}=0 \text { if } k=n \text { or } k+n \text { is even, } \\
\mathrm{D}_{\text {nk }}=-8 L k n / \pi^{2}\left(k^{2}-n^{2}\right)^{2} \text { if } k+n \text { is odd }
\end{gathered}
$$

Using this transition matrix $V_{k n}$ in Equation (20) and (21), we can find excitation/transition probability of any state at any time $t$ under chirped laser field. By using linear chirp, we will get some exciting new results which is unexpected by simple laser pulse. These are described in the results section.

\section{Computational Analysis}

The determination of coefficients $a_{n}(t)$ of different states allows us to calculate the transition probability for various laser pulse parameters. For the present calculations, we have studied the system with $n=10$ levels. Further, we have checked the convergence by variation in number of levels, and have obtained excellent convergence with $n=10$ levels.

For making the calculations traceable we have separated the real and imaginary parts of the coefficients $a_{n}(t)$ [see (18-21)]. Thus we obtain 20 real coupled differential equations to be solved. Any time propagator scheme can be used to solve these equations. For solving these equations we have used the efficient fourth order RungeKutta method by assuming that the system is initially in the ground state.

In next section, we discuss the results thus obtained. 


\section{Results}

It is worth mentioning, here, that we have used the box units throughout the paper. In this system of unit, the unit of mass is electron mass $\left(m_{e}\right)$, unit of length is $10^{-10} \mathrm{~m}$, unit of time is $1.75 \times 10^{-17} \mathrm{~s}$, unit of electric field is 3.75 $\times 10^{11} \mathrm{~V} / \mathrm{m}$ and unit of energy is ground state energy of electron in box of length $10^{-10} \mathrm{~m}$ i.e. $37.5 \mathrm{eV}$. Consider that initially the particle is in ground state i.e. $P_{1}(0)=1.0$. A plot of transition probability of particle in a box of length 2 box units as a function of time in optical cycle i.e. $t / \tau$ is shown in fig. 1 for zero chirp, positive chirp and negative chirp rates. Here laser center frequency is set at $\omega_{0}=3 / 4$ i.e. resonant frequency between $n=1$ and $n=2$ state, the laser field strength i.e. $E_{0}$ is $1 / 8$ and laser duration i.e. $\tau$ is 40.0 .
Figure 1 shows that transition probability of $n=2$ state is sufficiently large but there is some probability flow to higher states also and even there is probability flow to $n=10$ state. At the end of the pulse the transition probabilities becomes constant. It is clear from figure that the variations in transition probabilities are compressed and stretched with the help of chirp. The transition probabilities show oscillatory behavior during the pulse. The higher states shows more oscillatory behavior as compared to $n=1,2$ or 3 state.

In Figure 2 we have presented the variation of transition probability for different states as a function of laser chirp. The results here are taken at the end of the pulse. In this case central frequency $\omega_{0}$ has been taken as $1 / 8$ for $a, b$ and $3 / 8$ for $c, d$. Also laser duration $\tau$ is 40.0 for $a, d$ and 80.0 for $b, c$ subfigures. This is useful in com-

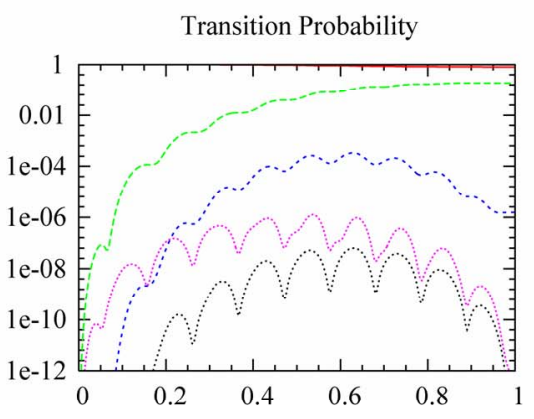

(a)

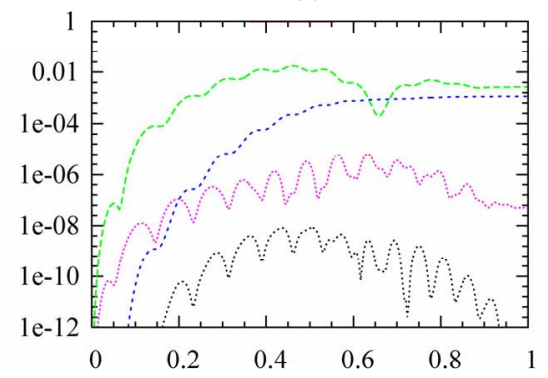

(d)

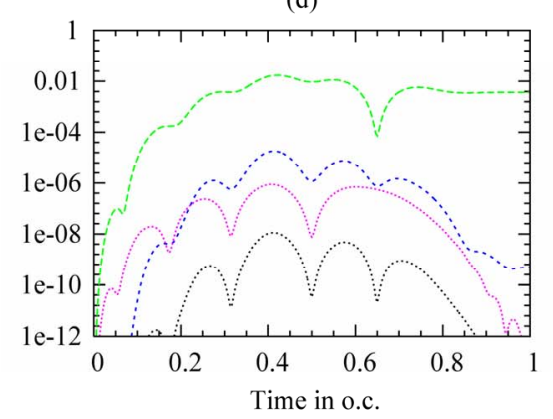

(g)

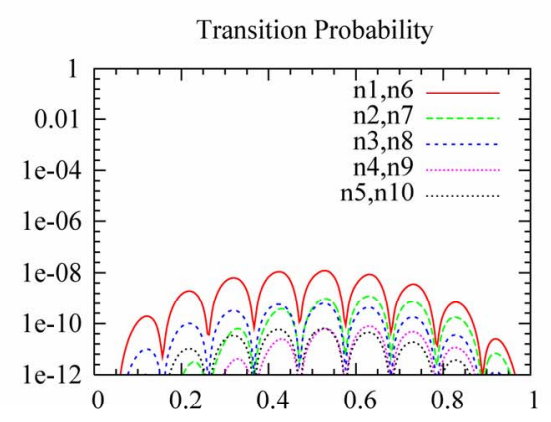

(b)

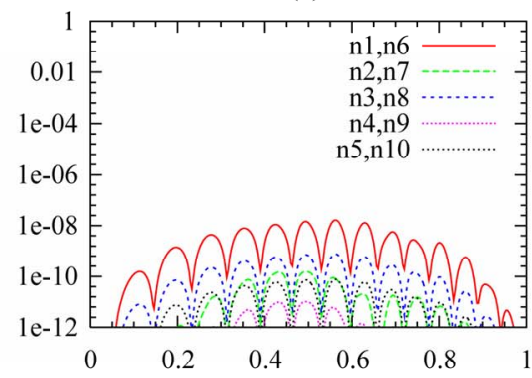

(e)

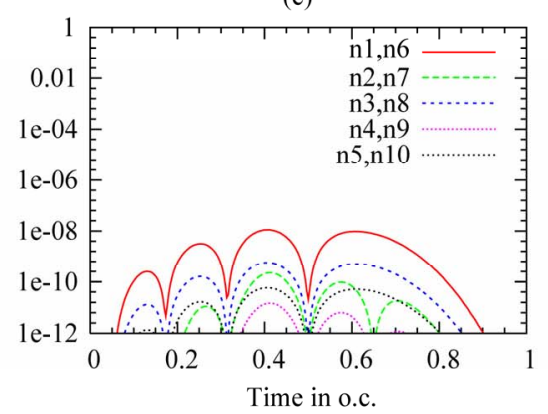

(h)

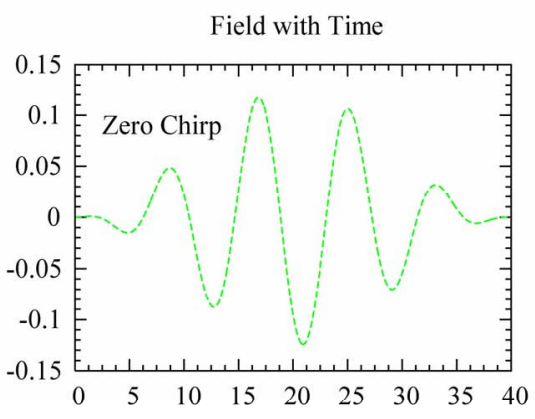

(c)

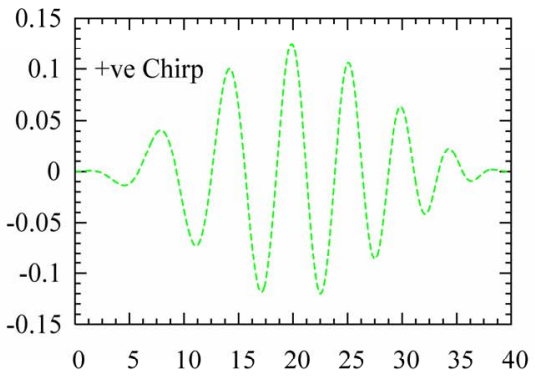

(f)

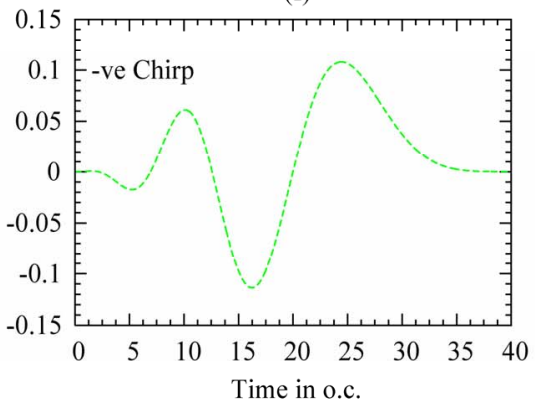

(i)

Figure 1. Transition probability of particle in a box in laser field as a function of time in optical cycle. Box width is $2 \times 10^{-10}$ $m$. Laser center frequency is $3 / 4$, laser duration $\tau=40$ and field amplitude $E_{0}=1 / 8$. In Figure $1(a, b, c) \beta=0.0$, Figure $1(d, e$, f) $\beta=+1.0 \times 10^{-2}$ and Figure $1(\mathrm{~g}, \mathrm{~h}, \mathrm{i}) \beta=-1.0 \times 10^{-2}$. Here, the key used explains that $n 1-n 5$ are for $a, d, g$ and $n 6-n 10$ are for $b, e, h$ subfigures. Subfigures $c, f, i$ refers to the corresponding fields. 


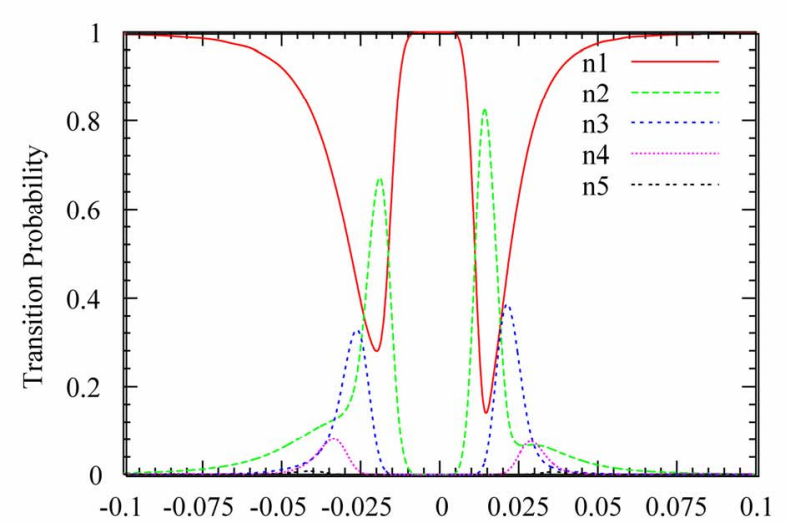

(a)

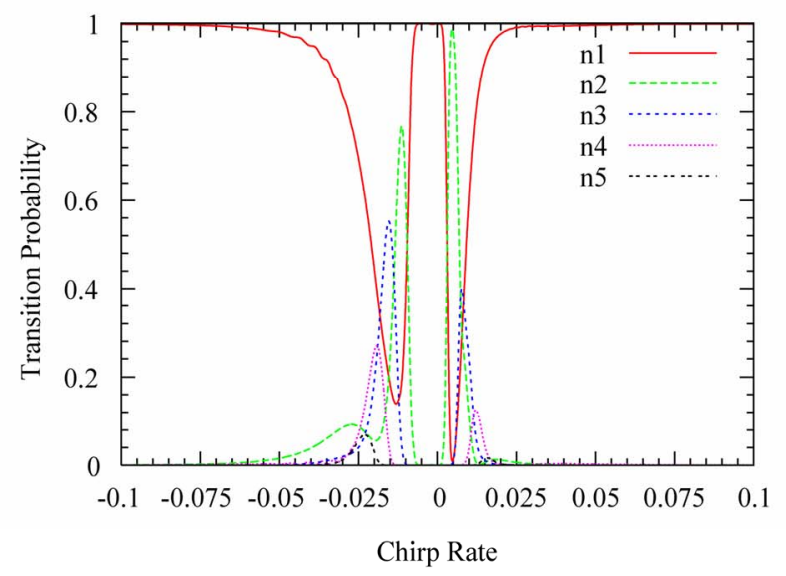

(c)

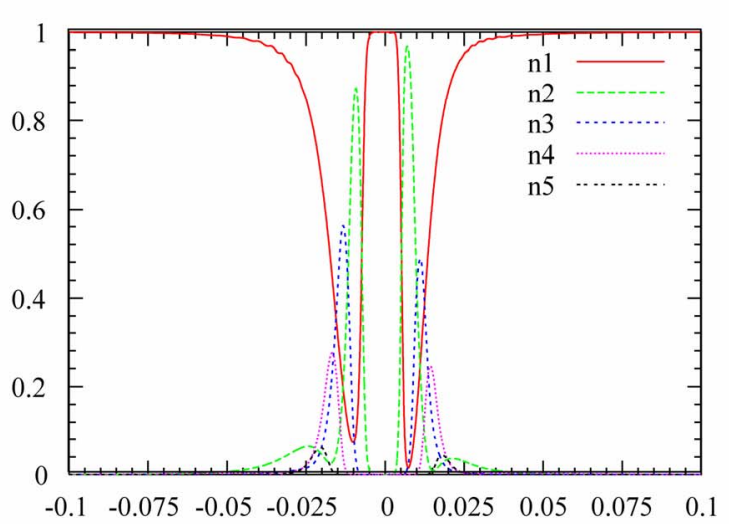

(b)

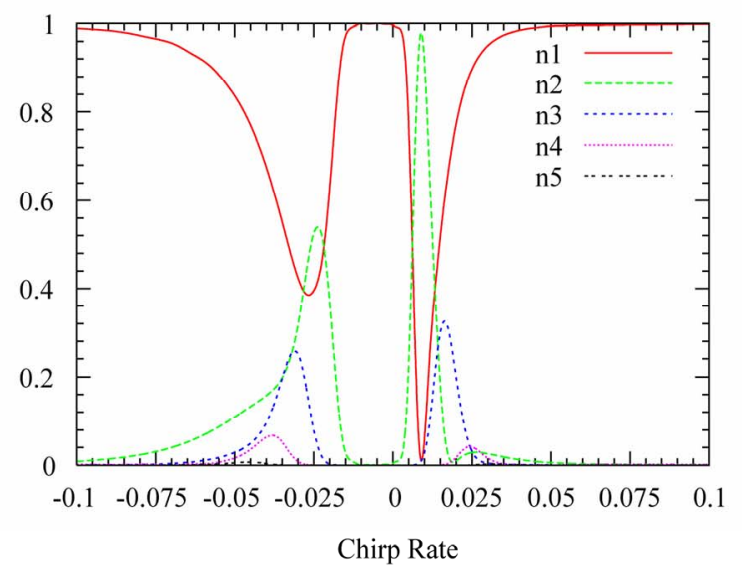

(d)

Figure 2. Transition probability for a particle in a box in chirped laser field as a function of chirp rate. Field strength $E_{0}=$ $1 / 2$ and box length $=2.0$. The centre frequency $\omega_{0}=1 / 8$ for $a, b$ and $\omega_{0}=3 / 8$ for $c, d$ subfigures. While the pulse width $\tau=$ 40.0 for $a, d$ and $\tau=\mathbf{8 0 . 0}$ for $b, c$ subfigures. The results are taken at the end of the pulse.

paring the results for variation of transition probability of different states as a function of chirp rate $(\beta)$ for two different frequencies and laser durations simultaneously. As it can be seen from figure that transition probabilities of $n=3$ state is higher for negative chirp than positive chirp for $\tau=40.0$ but it changes behviour for $\tau=80.0$, although transition probability for $\mathrm{n}=1$ and $\mathrm{n}=2$ states increases as frequency $\omega_{0}$ changes from $1 / 8$ to $3 / 8$ and transition probability remains greater for positive chirp than negative chirp. This change in probability shows that probability also depends on exposer time. Further, Figure 2 also shows that almost complete population inversion can be achieved by chirped laser pulses. There is substantial population transfer to higher states also.

Figure 3 represents the transition probability of 5 states as a function of box length. The results are taken at the end of the pulse. It is clear from the figure that negatively chirped pulse connects higher states more effecttively as compared to non-chirped pulse for the variation in box length. Also (d) part shows that transition probability of different states changes drastically for the exposed time.

In Figure 4, we compare the transition probability for 5 states as a function of laser frequency. The results are taken at the end of the pulse. In part (b) the resonance between $\mathrm{n}=1$ and $\mathrm{n}=2$ states occurs at their natural frequency but in part (a) the chirp shifts the resonance and also it connects the higher order terms more efficiently.

\section{Conclusions}

The effect of chirped laser pulse on particle in a box has been studied and we have come to the conclusion that frequency modulated laser pulse is an effective approach to get system in excited state. The transition probability of different states as a function of different pulse parameters and the box length have been studied. The sys- 


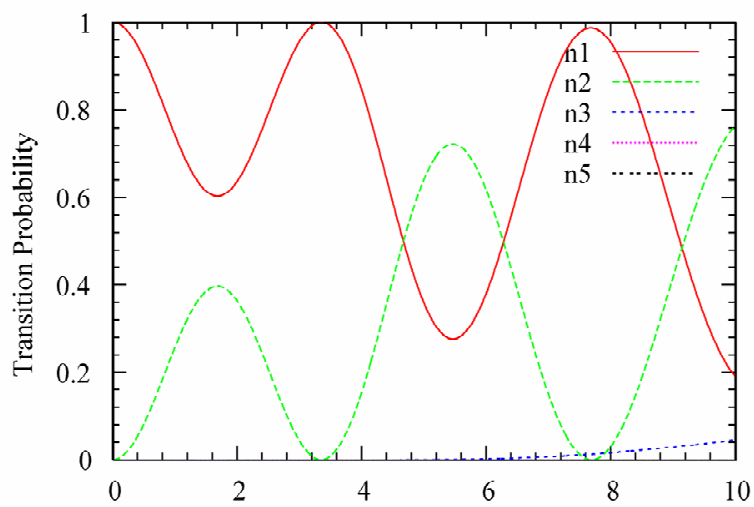

(a)

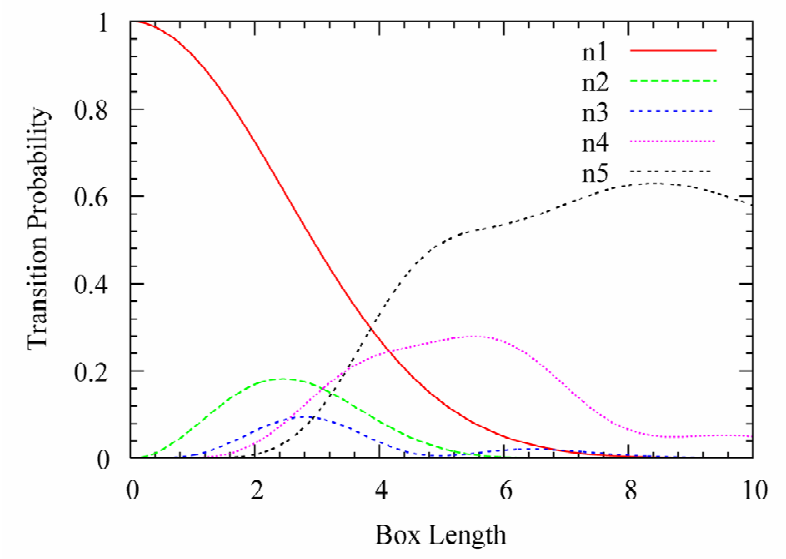

(c)

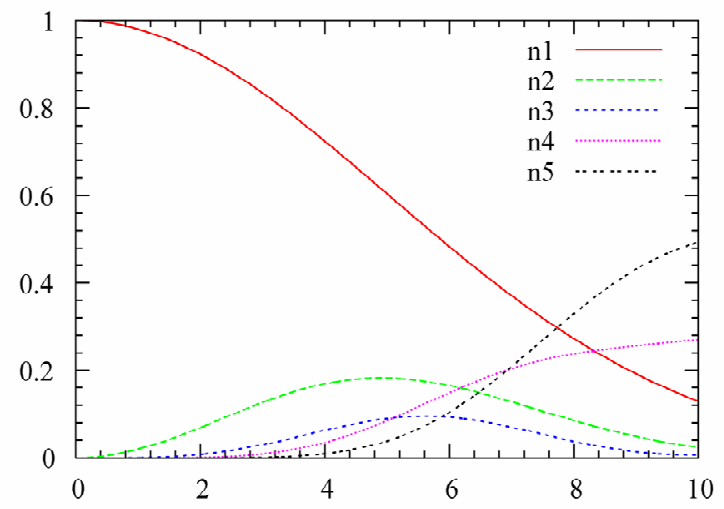

(b)

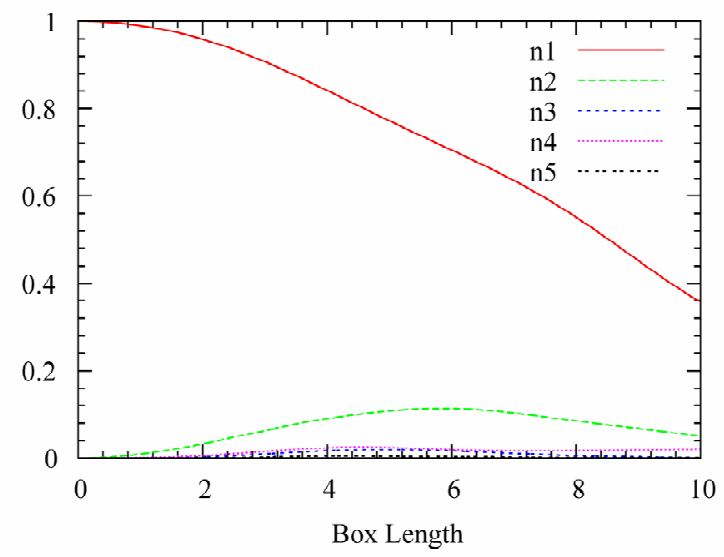

(d)

Figure 3. Transition probability of 5 states for particle in a box in chirped laser field as a function of box length. Chirp rate $\beta=-5.0 \times 10^{-2}$ (except for (a) where $\beta$ is zero), laser duration $\tau=40$ (except for (d) where $\tau$ is 80 ) and field amplitude $E_{0}=1 / 2$ (except for (b) where $E_{0}$ is $\left.1 / 4\right)$. The results are taken at the end of the pulse.

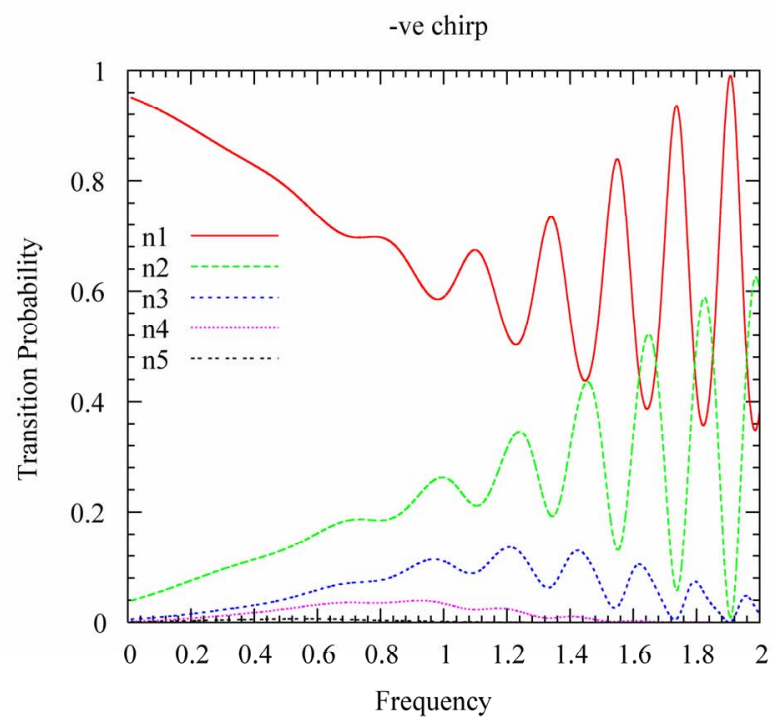

(a)

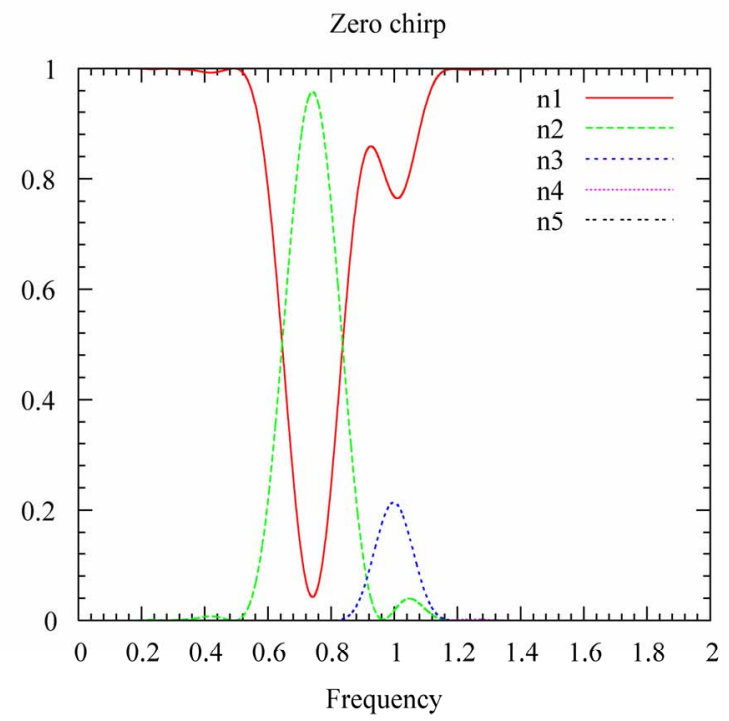

(b)

Figure 4. Transition probability of 5 states for particle in a box in chirped laser field as a function of central frequency. Chirp rate in part (a) is $\beta=-5.0 \times 10^{-2}$ and zero in part (b). Laser duration is $\tau=40$, box length is 2.0 and field amplitude $E_{0}=1 / 2$. The results are taken at the end of the pulse. 
tem is not merely two level shifting but with the help of chirped laser pulse we can shift the system to higher states, even $n=10$ state has some transition probability (whatever small, it may be). The main result of the study is the variation in the dynamics due to time varying external potential and large transition probabilities for higher states in the presence of chirped laser pulse which can't be achieved with non-chirped pulse. The system remains in higher state for appreciable time that may help in many physical and chemical aspects.

\section{Acknowledgements}

The authors are grateful to the unknown referee for valuable suggestions for the improvement of the paper.

\section{References}

[1] D. Bouwmeester, A. K. Ekert and A. Zeilinger, "The Physics of Quantum Information," Springer Berlin, NY, 2000.

[2] D. J. Griffiths, "Introduction to Quantum Mechanics," 2nd Edition, Pearson Prentice Hall, Upper Saddle River, New Jersey, 2005.

[3] J. Wang and J. D. Champagne, "Simulation of Quantum Systems with Coupled Channel Method," American Journal of Physics, Vol. 76, No. 4-5, 2008, pp. 493-497.

[4] W. Koechner, "Solid State Laser Engineering," 6th Edition, Springer, New York, 2006.

[5] H. Kawashima, M. M. Wefers and K. A. Nelson, "Fem- tosecond Pulse Shaping, Multiple-Pulse Spectroscopy and Optical Control," Annual Review of Physical Chemistry, Vol. 46, October 1995, pp. 627-656.

[6] P. Krehlik, "Characterization of Semiconductor Laser Frequency Chirp Based on Signal Distortion in Dispersive Optical Fiber," Opto-Electronics Review, Vol. 14, No. 2, June 2006, pp. 119-124.

[7] S. Ruhman and R. Kosloff, "Application of Chirped U1trashort Pulses for Generating Large-Amplitude GroundState Vibrational Coherence: A Computer Simulation,' Journal of the Optical Society of America B, Vol. 7, No. 8, August 1990, pp. 1748-1751.

[8] J. S. Cao, C. J. Bardeen and K. R. Wilson, "Molecular pulses: Population Inversion with Positively Chirped Short Pulses," Journal of Chemical Physics, Vol. 113, No. 5, August 2000, pp. 1898-1909.

[9] I. F. Barna and P. Dombi, "Coherent Control for the Spherical Symmetric Box Potential in Short and Intensive XUV Laser Fields," Central European Journal of Physics, Vol. 6, No. 2, June 2008, pp. 205-210.

[10] J. P. Hansen and K. Taulbjerg, "Coupled-Channel Calculations of Partial Capture Cross Sections in Multiply Charged Ion Collisions with Hydrogen," Physical Review A, Vol. 40, No. 7, October 1989, pp. 4082-4084.

[11] A. P. Hickman, "Coupled-Channel Calculations of Excited-Atom Collisions," International Reviews in Physical Chemistry, Vol. 16, No. 2, 1997, pp. 177-199.

[12] M. H. Mitleman "Introduction to the Theory of Laser Atom Interaction," Plenum Press, New York, 1982. 